\title{
Óleos essenciais no tratamento de dermatozoonoses - uma revisão de literatura
}

\author{
Essential oils in the treatment of dermatozoonoses - a literature review \\ Aceites esenciales en el tratamiento de la dermatozoonosis - una revisión de la literatura
}

\section{Resumo}

O uso indiscriminado de medicamentos tem representado uma das maiores ameaças à Saúde Única, haja vista sua atuação na resistência antimicrobiana e seus impactos ao meio ambiente. Assim, busca-se como alternativa sustentável a esses fármacos, os óleos essenciais, substâncias com diversas atividades terapêuticas, destacando a eficácia no tratamento de dermatopatias de origem zoonótica, a exemplo escabiose canina, esporotricose, dermatofitose e leishmaniose tegumentar americana. $O$ presente trabalho tem por objetivo realizar revisão bibliográfica acerca do emprego de óleos essenciais na medicina veterinária, destacando-se o tratamento das dermatopatias de origem zoonótica. Foi demonstrado o efeito acaricida, fungicida e fungistático dos óleos essenciais de Melaleuca alternifolia, Cedrus deodara, Azadirachta indica, Myrtus communis, Thymus vulgaris, Rosmarinus officinalis, Lavandula angustifolia, Cinnamomum cassia, Eugenia uniflora e Plectranthus amboinicus. As dermatozoonoses apresentam crescente resistência a antifúngicos e antibióticos comerciais, o emprego de terapias alternativas utilizando óleos essenciais se faz relevante de forma a amenizar essa resistência, e proporcionar melhoria na sanidade dos animais. Diante disso, dentre os estudos avaliados é demonstrado que os óleos essenciais de Melaleuca alternifolia, Cedrus deodara, Azadirachta indica, Myrtus communis, Thymus vulgaris, Rosmarinus officinalis, Lavandula angustifolia, Cinnamomum cassia, Eugenia uniflora e Plectranthus amboinicus apresentam resultado in vitro satisfatórios na terapêutica de dermatozoonoses de importância clínica, contudo, se faz necessário a realização de estudos in vivo para verificar a eficácia destes compostos, realizando-se um monitoramento de sua ação mediante as barreiras fisiológicas e metabolismo dos animais.

Palavras-chave: Resistência antimicrobiana; Medicamentos fitoterápicos; Dermatopatias. 


\begin{abstract}
The indiscriminate use of medications has represented one of the greatest threats to Unique Health, given its role in antimicrobial resistance and its impacts on the environment. Thus, essential oils, substances with various therapeutic activities, are sought as a sustainable alternative to these drugs, highlighting their effectiveness in the treatment of dermatopathies of zoonotic origin, such as canine scabies, sporotrichosis, dermatophytosis and American tegumentary leishmaniasis. The present work aims to carry out a literature review on the use of essential oils in veterinary medicine, highlighting the treatment of dermatopathies of zoonotic origin. The acaricide, fungicidal and fungistatic effect of essential oils from Melaleuca alternifolia, Cedrus deodara, Azadirachta indica, Myrtus communis, Thymus vulgaris, Rosmarinus officinalis, Lavandula angustifolia, Cinnamomum cassia, Eugenia uniflora and Plectranthus amboinicus was demonstrated. Dermatozoonosis is increasingly resistant to commercial antifungals and antibiotics, and the use of alternative therapies using essential oils is relevant in order to alleviate this resistance and improve the health of animals. Therefore, among the evaluated studies, it is demonstrated that the essential oils of Melaleuca alternifolia, Cedrus deodara, Azadirachta indica, Myrtus communis, Thymus vulgaris, Rosmarinus officinalis, Lavandula angustifolia, Cinnamomum cassia, Eugenia uniflora and Plectranthus amboinicus present satisfactory therapeutic results in vitro of clinically important dermatozoonoses, however, it is necessary to carry out in vivo studies to verify the effectiveness of these compounds, monitoring their action through physiological barriers and metabolism of animals.
\end{abstract}

Keywords: Antimicrobial resistance; Herbal medicines; Dermatopathies.

\title{
Resumen
}

El uso indiscriminado de medicamentos ha representado una de las mayores amenazas para Unique Health, dado su papel en la resistencia a los antimicrobianos y sus impactos en el medio ambiente. Así, se buscan aceites esenciales, sustancias con diversas actividades terapéuticas, como alternativa sostenible a estos fármacos, destacando su eficacia en el tratamiento de dermatopatías de origen zoonótico, como sarna canina, esporotricosis, dermatofitosis y leishmaniasis tegumentaria americana. El presente trabajo tiene como objetivo realizar una revisión de la literatura sobre el uso de aceites esenciales en medicina veterinaria, destacando el tratamiento de las dermatopatías de origen zoonótico. Se demostró el efecto acaricida, fungicida y fungistático de los aceites esenciales de Melaleuca alternifolia, Cedrus deodara, Azadirachta indica, Myrtus communis, Thymus vulgaris, Rosmarinus officinalis, Lavandula angustifolia, Cinnamomum cassia, Eugenia uniflora y Plectranthus amboinicus. La dermatozoonosis es cada vez más resistente a los antifúngicos y antibióticos comerciales, y el uso de terapias alternativas con aceites esenciales es relevante para paliar esta resistencia y mejorar la salud de los animales. Por tanto, entre los estudios evaluados, se demuestra que los aceites esenciales de Melaleuca alternifolia, Cedrus deodara, Azadirachta indica, Myrtus communis, Thymus vulgaris, Rosmarinus officinalis, Lavandula angustifolia, Cinnamomum cassia, Eugenia uniflora y Plectranthus amboinicus presentan resultados terapéuticos in vitro satisfactorios. de dermatozoonosis clínicamente importantes, sin embargo, es necesario realizar estudios in vivo para verificar la efectividad de estos compuestos, monitoreando su acción a través de barreras fisiológicas y el metabolismo de los animales.

Palabras clave: Resistencia a los antimicrobianos; Hierbas medicinales; Dermatopatías.

\section{Introdução}

A resistência aos antimicrobianos é, atualmente, uma das ameaças à Saúde Única mais relevantes (Adams et al., 2018; Hernandez, 2017). O uso indiscriminado, errôneo e abusivo de antimicrobianos, tanto na medicina humana quanto na veterinária, tem elevado as taxas de resistência aos antibióticos, antifúngicos e antiparasitários, criando organismos multirresistentes, o que limita as opções de tratamento e trazem consequências clínicas e econômicas graves, contribuindo com o aumento da morbimortalidade em humanos e animais (Loureiro et al., 2016). Dessa forma a profilaxia e tratamento de doenças infecciosas tem se tornado um desafio para os profissionais da saúde pois os fármacos antimicrobianos eficazes se tornam cada vez mais escassos.

Outra problemática envolvendo o uso de antimicrobianos é o seu descarte inadequado que pode provocar desequilíbrio ambiental através da contaminação dos lençóis freáticos, com consequente comprometimento da fauna e da flora. Além disso, as águas residuais de estações de tratamento podem atuar como potencial reservatório de patógenos resistentes (Karkman et al., 2017; Ueda et al., 2009). Diversos artigos científicos revelam a ocorrência de microrganismos multirresistentes em animais silvestres, o que demonstra a expansão do problema (Gaio et al., 2019).

Em paralelo a este cenário, um número reduzido de novas classes de drogas antibacterianas foi descoberto nas últimas décadas, por essa razão, a busca por novos metabólitos ativos e alternativas contra patógenos resistentes se tornou necessária. 
Embora maior ênfase seja dada a resistência bacteriana aos antimicrobianos, é importante salientar que muitos fungos, em especial os dermatófitos, têm demonstrado resistência a desinfetantes e antifúngicos convencionais (Ceconi et al., 2018; Avante et al., 2009).

Derivados de produtos de origem vegetal, como óleos essenciais, que trazem menos impactos negativos à saúde animal, ambiental e humana têm sido pesquisados. Os óleos essenciais são substâncias voláteis derivadas do metabolismo secundário de plantas, com propriedades antimicrobianas, anti-inflamatórias, antiparasitárias e antipruriginosas. São utilizados para diversos fins, a exemplo na produção agrícola, química, farmacêutica e também na indústria alimentícia (Bell, 2002; Lupe, 2007; Cossolosso, 2013).

Na medicina veterinária os óleos essenciais são amplamente utilizados nas práticas de aromaterapia. Trata-se de tratamento holístico realizado através de aromas de partículas que atuam sobre o sistema nervoso de forma a proporcionar estímulos ou calmaria (Bell, 2002). O uso dos óleos essenciais tem sido adotado como medidas terapêuticas nas dermatopatias zoonóticas, que são enfermidades de extrema importância no âmbito de Saúde Única (Mateus, 2016; Faria, 2011; Baptista, 2015).

As dermatopatias podem ser de origem bacteriana, parasitária e fúngica. Na clínica médico-veterinária as dermatites fúngicas possuem maior destaque, sobretudo as dermatofitoses (Paterson, 2008; Hnilica, 2011) e a esporotricose, além de dermatites parasitárias, como a escabiose canina, esporotricose e a leishmaniose tegumentar americana. São doenças de manifestação majoritariamente cutânea, associadas ao risco de transmissão aos humanos e animais (Canavari et al., 2017). Assim, diversos estudos têm sido realizados em busca de uma terapêutica alternativa com o uso de óleos essenciais frente a essas enfermidades, visando avaliar a efetividade desses elementos em relação à inibição ou destruição dos agentes causadores dessas patologias.

Dessa forma, com o intuito de facilitar a disseminação de conhecimento e auxiliar na profilaxia e condução do tratamento alternativo dessas enfermidades, o presente trabalho busca revisar as principais utilidades dos óleos essenciais na medicina veterinária, com ênfase no tratamento de dermatopatias de origem zoonótica.

\section{Metodologia}

Para esquematização da revisão integrativa de literatura foi realizada a inclusão da pesquisa qualitativa, onde foram analisados vários trabalhos com o intuito de fornecer os parâmetros necessários para uma avaliação criteriosa acerca dos dados científicos disponíveis frente a aplicabilidade dos óleos essenciais em animais. Para essa análise foi designada a pergunta científica, realização de pesquisa bibliográfica, análise e avaliação dos dados e apresentação dos resultados através da produção do artigo de revisão. As bases de dados utilizadas para a pesquisa foram: Scientific Electronic Library Online SciELO, Google Acadêmico e Aromatic Science, além de livros, dissertações e teses. A busca da literatura foi realizada entre os meses de agosto e dezembro de 2020, utilizando descritores como "dermatozoonoses", "aromaterapia" e "óleos essenciais", que facilitam o acesso às bibliografias (Figura 1). 
Figura 1: Fluxograma de etapas desenvolvidas para elaboração do artigo de revisão.

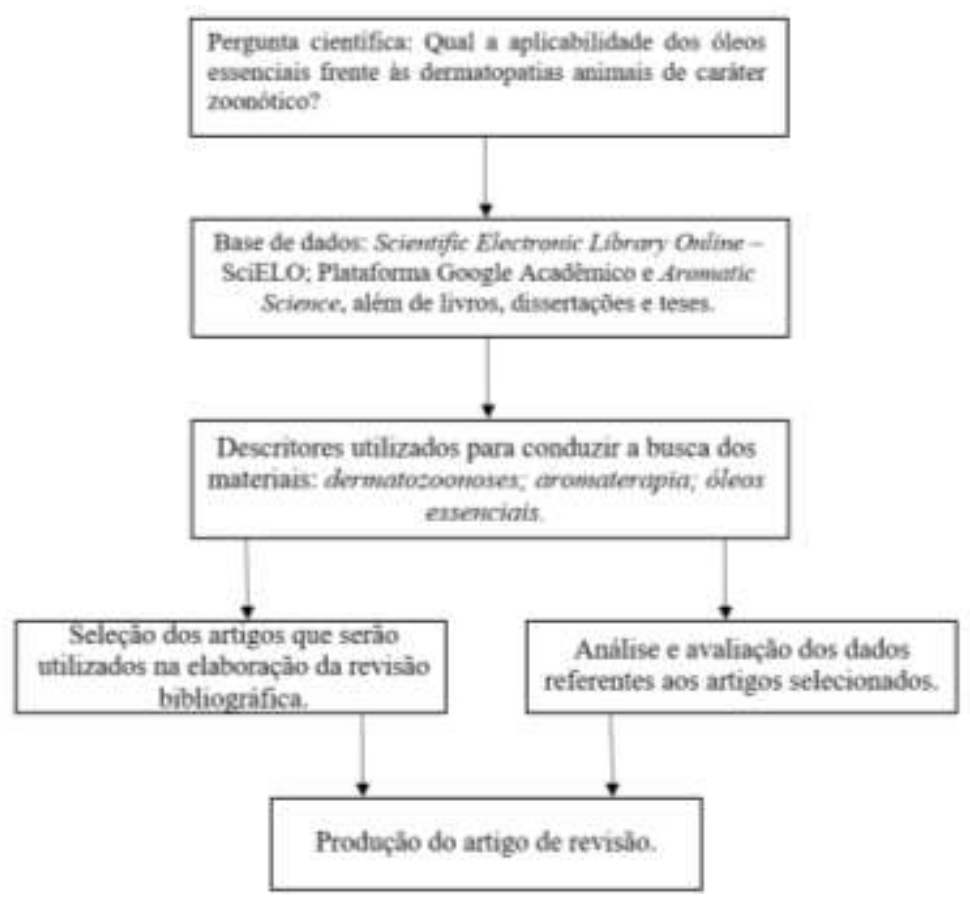

Fonte: Arquivo pessoal.

A busca, seleção e avaliação dos estudos foi realizada de forma simultânea pelos pesquisadores. Estes trabalhos, através da leitura do título e resumo, foram submetidos aos critérios de inclusão: artigos originais, que descrevessem a atividade dos óleos essenciais frente às dermatozoonoses; artigos publicados em periódicos de saúde pública escritos por autores renomados na área de estudo proposta, completos e com publicação recente, além de teses de mestrado e doutorado. E aos critérios de exclusão: artigos repetidos, manual, editorial, resenha, carta, protocolos de estudo e diretrizes de cuidado. Em seguida, os artigos escolhidos foram direcionados para uma avaliação minuciosa, onde foram realizadas comparações e análises reflexivas com discussão embasada pela literatura disponível.

Foram selecionados um total de 34 artigos que entraram no corpo da revisão, envolvendo artigos nacionais e internacionais (Tabela 1). 
Tabela 1. Artigos, dissertações e teses utilizados para elaboração da revisão.

\begin{tabular}{|c|c|c|}
\hline Nome dos artigos/ dissertações/ teses/ livros & Autores & $\begin{array}{c}\text { Ano de } \\
\text { publicação }\end{array}$ \\
\hline $\begin{array}{l}\text { Neem seed extract shampoo, Wash Away Louse®, an effective plant agent against Sarcoptes scabiei mites } \\
\text { infesting dogs in Egypt. }\end{array}$ & Abdel-Ghaffar, F. et al & 2008 \\
\hline Antimicrobial-resistant Enterobacteriaceae recovered from companion animal and livestock environments. & Adams, R. J. et al & 2018 \\
\hline Sarna sarcóptica em cães: uma breve revisão. & Almeida, L. C. et al & 2019 \\
\hline One health - a saúde única sob a percepção do estudante de medicina veterinária do Distrito Federal. & Araujo, S. A. et al & 2020 \\
\hline Resistência antimicrobiana nos animais e no ser humano. Há motivo para preocupação?. & Arias, M. V. B. et al & 2012 \\
\hline Estudo de estabilidade e eficácia de formulação tópica fitoterápica para tratamento de dermatofitose animal. & Baptista, B. E. & 2015 \\
\hline $\begin{array}{l}\text { Holistic Aromatherapy for Animals: A Comprehensive Guide to the Use of Essential Oils e Hydrosols with } \\
\text { Animals. }\end{array}$ & Bell, K. L. & 2002 \\
\hline Efeitos de óleos essenciais como promotores de crescimento em leitões recém desmamados. & Branco, P. A. C. et al & 2011 \\
\hline Doenças Dermatológicas de Caráter Zoonótico. & Canavari, I. C. et al & 2017 \\
\hline $\begin{array}{l}\text { Trajetória do projeto de extensão: "Medicina Veterinária na promoção da saúde humana e animal: Ações } \\
\text { em comunidades carentes como estratégia para o enfrentamento da desigualdade social". }\end{array}$ & Cleff, B. M. et al & 2020 \\
\hline Atividades leishmanicida e antioxidante dos óleos essenciais de plantas encontradas no Nordeste brasileiro. & Cossolosso, D. S. & 2013 \\
\hline Esporotricose: Revisão. & $\begin{array}{l}\text { Cavalcanti, D. L. N. A. E } \\
\text { et al }\end{array}$ & 2018 \\
\hline In vitro activity of ten essential oils against Sarcoptes scabiei. & Fang, F. et al & 2016 \\
\hline Tratamentos convencionais e fitoterápicos para o controle de sarna sarcóptica nos animais domésticos. & Faria, A. M. & 2020 \\
\hline Aditivos fitopatogênicos na alimentação de frangos de corte: óleos essenciais e especiarias. & Fernandes, V. T. R. et al & 2015 \\
\hline $\begin{array}{l}\text { Aspectos clínicos, diagnósticos e terapêuticos da dermatofitose em cães e gatos e sua importância como } \\
\text { zoonose. }\end{array}$ & $\begin{array}{l}\text { Gondim, L. C. L. A. \& } \\
\text { Araujo, L. K. A. }\end{array}$ & 2020 \\
\hline Biodiversidade. Um enfoque químico - biológico. & Gottlieb, O. R. et al & 1996 \\
\hline $\begin{array}{l}\text { Bactérias zoonóticas isoladas de passeiriformes silvestres recuperados do tráfico de animais no estado do } \\
\text { Ceará/Brasil. }\end{array}$ & $\begin{array}{l}\text { Gaio, F. C. \& Maciel, W. } \\
\text { C. }\end{array}$ & 2019 \\
\hline Impacto do uso de antimicrobianos na medicina veterinária. & Hernandez, B. J. et al & 2017 \\
\hline Antibiotic-Resistance Genes in WasteWater. & Karkman, A. T. T. et al & 2018 \\
\hline $\begin{array}{l}\text { Atividade leishmanicida do óleo essencial de Siparuna guianensis e do isolado } \alpha \text {-Bisabolol isolado de } \\
\text { Siparuna guianensis contra Leishmania amazonensis. }\end{array}$ & Lima, A. P. L. & 2018 \\
\hline Óleos essenciais como alternativa inovadora para o tratamento da esporotricose. & Lima, R. M. & 2017 \\
\hline O uso de antibióticos e as resistências bacterianas: breves notas sobre a sua evolução. & Loureiro, R. J. & 2016 \\
\hline Estudo da composição química de óleos essenciais de plantas aromáticas da Amazônia. & Lupe, F. A. & 2020 \\
\hline $\begin{array}{l}\text { Avaliação dos efeitos fungistáticos e fungicidas de óleos essenciais em microrganismos causadores de } \\
\text { dermatomicoses. }\end{array}$ & Mateus, W. S. & 2016 \\
\hline Aromatherapy for parrots: using ancient healing methods in today’s companion birds. & Nelson, A. & 2013 \\
\hline Presença de dermatófitos em animais infectados e seu ambiente doméstico. & Neves, J. J. et al & 2018 \\
\hline $\begin{array}{l}\text { Desenvolvimento de uma emulsão o/a associada ao óleo essencial de gerânio (Pelargonium graveolens) e } \\
\text { ao óleo essencial de palmarosa (Cymbopogon martinii). }\end{array}$ & $\begin{array}{l}\text { Oliveira, S. \& Morais, P. } \\
\text { A. C. }\end{array}$ & 2019 \\
\hline $\begin{array}{l}\text { Atividade antimicrobiana de óleos essenciais de condimentos frente a Staphylococcus spp isolados de } \\
\text { mastite caprina. }\end{array}$ & Pozzo, M. D. et al & 2011 \\
\hline Atividade leishmanicida in vitro de Eugenia uniflora e Mormodica charantia. & Santos, K. K. A. et al & 2013 \\
\hline Isolamento, Identificação e Atividade Antimicrobiana de Streptomyces sp. & Soares, E. C. L. & 2012 \\
\hline $\begin{array}{l}\text { Impacto Ambiental do Descarte de Fármacos e Estudo da Conscientização da População a Respeito do } \\
\text { Problema. }\end{array}$ & Ueda, J. & 2009 \\
\hline Potencial anti-Sporothrix spp. de plantas da família lamiaceae. & Waller, S. B. & 2015 \\
\hline $\begin{array}{l}\text { Effects of essential oils of Rosmarinus officinalis Linn. and Origanum vulgare Linn. from different origins } \\
\text { on Sporothrix brasiliensis and Sporothrix schenckii complex. }\end{array}$ & Waller, S. B. et al & 2016 \\
\hline
\end{tabular}

Fonte: Arquivo pessoal.

A etapa final do processo de revisão bibliográfica constituiu-se da exposição dos dados analisados de forma descritiva. Dessa forma, os resultados e conclusão da revisão em questão estão sintetizados conforme os objetivos do estudo.

\section{Resultados e Discussão}

\subsection{História e composição dos óleos essenciais}

Das cerca de 250 mil espécies de plantas no mundo apenas $10 \%$ foram testadas para algum tipo de atividade biológica (Soares et al., 2012). Os levantamentos florísticos e os estudos etnobotânicos são muito importantes no Brasil, visto que seu território abriga uma das mais ricas floras do mundo, embora apenas $0,4 \%$ sejam quimicamente conhecidas. Na região Nordeste do país encontram-se mais de 10 mil espécies, aproximadamente 50\% desses exemplares pertencentes a vegetação de caatinga e cerrado (Gottlieb et al., 1996). 
A utilização de plantas para fins terapêuticos é uma prática tão antiga quanto a história da humanidade e dentre os seus derivados, destaca-se os óleos essenciais, que são substâncias líquidas aromáticas com ação antimicrobiana, e seus hidrolatos, que se diferenciam por terem propriedades diluídas. Os testes com óleos essenciais ocorreram inicialmente em animais para uso posterior em humanos, com início na década de 80, contudo, o desenvolvimento da prática terapêutica desses elementos na medicina veterinária iniciou-se alguns anos depois, já na década de 90 (Bell, 2002).

Diversas técnicas podem ser usadas na extração dos óleos essenciais, dentre elas o "enfleurage", arraste por vapor d'água, extração com solventes orgânicos, prensagem (ou expressão) e extração por $\mathrm{CO} 2$ supercrítico. Estudos anteriores demonstraram que seus principais constituintes, de forma geral, são os derivados de fenilpropanóides e terpenóides. Sabe-se também que sua composição química pode ser alterada devido a fatores ambientais (Lupe, 2007).

Para comprovar o potencial danoso dos óleos essenciais é feito o teste de estabilidade, que é medido pelo conjunto de práticas que possibilitam comprovar efeitos benéficos e descartar possíveis danos ao organismo dos indivíduos. Nesse teste os óleos são expostos a condições climáticas controladas e distintas evitando assim que o produto seja submetido a situações estressantes que consequentemente alteram sua eficiência. A estabilidade do produto pode ser afetada tanto por fatores extrínsecos a exemplo da luz, temperatura, umidade, calor, tempo, oxigênio, contaminação microbiológica e vibração, como também por fatores intrínsecos associados às suas propriedades físico-químicas (Baptista, 2015), bem como as características organolépticas como odor, cor e ph são determinantes para aprovação ou rejeição do produto pela Agência Nacional de Vigilância Sanitária - ANVISA (Oliveira \& Morais, 2019). Adicionalmente deve-se considerar questões de estabilidade e toxicidade para o emprego em animais domésticos

Quanto ao mecanismo de ação, em geral, os óleos essenciais agem alterando o potencial de membrana da célula, afetando o pH e a síntese de adenosina trifosfato (ATP). Além disso, por terem afinidade e serem solúveis em lipídios, causam danos também à membrana plasmática de células fúngicas (Baptista, 2015).

\subsection{Importância na Saúde Única}

A utilização dos óleos essenciais na medicina veterinária contribui amplamente para a promoção da Saúde Única, tendo em vista que sua aplicação na terapêutica veterinária pode prevenir o surgimento de resistência antimicrobiana, ou tornar-se-á uma possibilidade de tratamento de afecções ocasionadas por patógenos resistentes. Além disso, o emprego da aromaterapia pode ter consequências na diminuição dos impactos negativos que o descarte inadequado dos antibióticos proporciona ao ambiente. (Arias \& Carrilho, 2012; Ueda et al., 2009).

\subsubsection{Esporotricose}

A esporotricose é uma enfermidade com elevado potencial zoonótico causada por fungos do complexo Sporothrix schenckii. Os felinos, em especial os da espécie Felis catus, são os principais afetados e os maiores transmissores. Quanto ao emprego de óleos essenciais, Lima (2017) avaliou in vitro a atuação de Myrtus communis (mirta) e Thymus vulgaris (tomilho) diante das linhagens fúngicas Sporothrix schenckii ATCC 1099-18 e Sporothrix schenckii IPEC 15383. O óleo de mirta demonstrou efeito sobre a capacidade de inibir o crescimento fúngico com concentrações que variaram entre 31,25 a 62,5 $\mu \mathrm{g} / \mathrm{mL}$ frente às duas linhagens. Em relação à ação fungicida, também foi observada em todas as linhagens, com concentração acima de $62,5 \mu \mathrm{g} / \mathrm{mL}$. Já o óleo de tomilho promoveu a inibição do crescimento em todas as linhagens, mas com concentração entre 125 a $250 \mu \mathrm{g} / \mathrm{mL}$, e atividade fungicida em ambas, porém a concentração ultrapassou $250 \mu \mathrm{g} / \mathrm{mL}$. Ademais, foi observado indícios de resistência fúngica, visto que medicamentos utilizados convencionalmente como anfotericina $\mathrm{B}$ e itraconazol demonstraram eficácia parcial ou nula, respectivamente, em relação à susceptibilidade das linhagens avaliadas. 
Em contrapartida, Waller et al. (2016) relataram os resultados encontrados por meio da experimentação dos óleos essenciais de Rosmarinus officinalis L. (alecrim) e Origanum vulgare L. (orégano), comerciais e naturais, diante de isolados de Sporothrix schenckii coletados de humanos, cães, felinos e também do ambiente, onde foram descritos os efeitos dessas substâncias e principais constituintes. Ambos os óleos se mostraram eficazes nos resultados in vitro. Em relação a composição, o óleo de alecrim apresentou como principal componente o 1,8-cineol para os óleos obtidos de ambas as formas, enquanto que o óleo essencial de orégano demonstrou variações, sendo encontrado como componente majoritário o timol no óleo extraído e o carvacrol no óleo comercial. Esses compostos são os responsáveis pela atividade anti-Sporothrix. Com isso, há a indicação de um possível tratamento promissor para a esporotricose, que deve ser testado in vivo, para comprovação de sua eficácia mediante as condições fisiológicas de cada hospedeiro.

\subsubsection{Dermatofitoses}

As dermatofitoses são enfermidades cutâneas altamente contagiosas, consideradas zoonoses de fundamental importância em Saúde Única. Podem ser transmitidas através do contato direto com animais, humanos ou solos infectados, ou de forma indireta, por meio de fômites contaminados (Neves et al., 2018). São causadas por dermatófitos, fungos filamentosos que possuem atração por áreas queratinizadas. Estes, são pertencentes a três gêneros: Microsporum, Trichophyton e Epidermopyton (Gondim \& Araujo, 2020), sendo Microsporum canis, Microsporum gypseum e Trichophyton mentagrophytes as espécies mais frequentes (Canavari et al., 2017).

Baptista (2015) expõe a capacidade antifúngica dos óleos essenciais de Pimenta pseudocaryophyllus (craveiro) e Eucalyptus smithii (eucalipto) perante os fungos Trichophyton mentagrophytes ATCC 11480, Microsporum canis ATCC 32903 e Microsporum gypseum ATCC 14683. Pôde-se analisar após obtenção dos resultados experimentais que os óleos derivados da Pimenta pseudocaryophyllus foram mais eficazes. Além disso, os estudos de estabilidade e eficácia de formulação de cremes com base no óleo essencial dessa última se mostraram promissores.

Mateus (2016) relata que na avaliação fungistática e fungicida dos óleos essenciais de Rosmarinus officinalis (alecrim), Lavandula angustifolia (alfazema), Cinnamomum cassia (canela) e Melaleuca alternifolia (Melaleuca) frente às espécies fúngicas Trichophyton rubrum ATCC® MYA 4438, Trichophyton mentagrophytes var. interdigitale ATCC® MYA 4439 e Trichophyton mentagrophytes, o óleo essencial de alecrim não apresentou atividade considerável, o de alfazema e melaleuca inibiram o crescimento fúngico, e o de canela demonstrou maior eficiência, com efeito fungicida sobre todos os fungos.

\subsubsection{Leishmaniose Tegumentar Americana}

A leishmaniose é uma infecção zoonótica que representa grande risco a saúde pública. Sua forma tegumentar possui como características clínicas predominantes lesões, caspas e feridas com crostas no tecido cutâneo. É causada por protozoários do gênero Leishmania e sua transmissão ocorre através de vetores, como flebotomíneos do gênero Lutzomyia. Os cães são considerados como principais reservatórios em centros urbanos, e os humanos são tidos como hospedeiros acidentais.

O trabalho realizado por Cossolosso (2013) avaliou a ação in vitro dos óleos essenciais de Eugenia uniflora (pitanga), Plectranthus amboinicus (hortelã-grosso) e de espécies de Crotons sp., onde todos apresentaram atividade leishmanicida. Santos et al. (2013) realizaram testes in vitro com o óleo de Eugenia uniflora em relação às cepas de parasitas, obtendo resultados positivos. Lima (2018) descreveu o potencial também in vitro do óleo de Siparuna guianensis e do seu composto isolado $\alpha$-Bisabolol, frente a Leishmania amazonenses, onde houve inibição em $100 \%$ do crescimento do parasito na maioria das concentrações testadas, ressaltando assim a importância de estudos mais aprofundados para posterior testagem in vivo. 


\subsubsection{Sarna Sarcóptica}

O ácaro Sarcoptes scabiei é causador de uma importante dermatopatia na medicina veterinária, denominada de sarna sarcóptica, apresentando relevância destacada na clínica de pequenos animais, em razão do seu elevado número de casos (Almeida, 2019). Caracterizada por provocar lesões pruriginosas que afetam sobretudo a camada superficial da pele, possui o cão doméstico como principal espécie acometida, este pode atuar como fonte de transmissão aos seres humanos.

Considerando a necessidade de controlar e combater essa patogenia Fang et al. (2016) avaliaram dez óleos essenciais: Lavandula angustifolia (lavanda), Citrus aurantium amara (laranja amarga), Pelargonium asperum (Gerânio), Melaleuca alternifolia (tea tree), Syzygium aromaticum (cravo), Eucalyptus radiata (eucalipto), Leptospermum scoparium (manuka), Juniperus oxycedrus (cade), Cryptomeria japonica (cedro japonês) e Cymbopogon martini (palmarosa), frente aos ácaros Sarcoptes scabiei previamente colhidos de suínos infectados para experimentação in vitro . Conforme a conclusão da pesquisa, foi possível analisar que os óleos mais eficazes são os de cravo, eucalipto, melaleuca e palmarosa, sendo o óleo de cade o único a não apresentar nenhum resultado durante a realização dos testes.

Abdel-Ghaffar et al. (2008) demonstraram o potencial antiparasitário do óleo extraído das sementes de Azadirachta indica (neem) através do tratamento realizado com a aplicação tópica do shampoo derivado dessa substância em dez cães portadores da sarna sarcóptica, observando redução dos parasitas e melhora do quadro clínico desses animais. Faria (2011) retrata os resultados obtidos com a utilização e análise de três óleos essenciais diante dos agentes etiológicos da escabiose animal. Dentre eles, pode-se citar o óleo proveniente da Melaleuca alternifolia, que na concentração de 5\% apresentou eficácia contra os ácaros, fator que pode ser explicado pela presença de compostos antimicrobianos como os terpenos. O óleo essencial de Cedrus deodara (cedro do himalaia) também possui o terpeno na sua composição, sendo um dos responsáveis pela atividade acaricida. Extraído da Azadirachta indica, o óleo de neem também possui efetividade comprovada contra ácaros, e o elemento responsável por essa ação é a azaractina.

Com base nos estudos analisados a respeito do uso dos óleos essenciais na terapêutica da esporotricose, dermatofitose, leishmaniose e sarna sarcóptica, depreende-se, portanto, a necessidade de estudos in vivo abordando o uso dos óleos essenciais no tratamento de dermatozoonoses em animais domésticos, em especial os pequenos animais, tendo em vista a segurança que as substâncias mencionadas apresentam e o alto grau de contágio dos agentes causadores das dermatozoonoses.

\section{Conclusão}

As dermatozoonoses apresentam crescente resistência antifúngica e o emprego de terapias alternativas se faz relevante de forma a amenizar o desconforto do animal ao eliminar o agente causador. Diante disso, dentre os estudos avaliados identifica-se que os óleos essenciais de Melaleuca alternifolia, Cedrus deodara, Azadirachta indica, Myrtus communis, Thymus vulgaris, Rosmarinus officinalis, Lavandula angustifolia, Cinnamomum cassia, Eugenia uniflora e Plectranthus amboinicus apresentam resultado in vitro satisfatórios na terapêutica de dermatozoonoses de importância clínica, contudo, se faz necessário a realização de estudos in vivo para verificar a eficácia destes compostos, realizando-se um monitoramento de sua ação mediante as barreiras fisiológicas e metabolismo dos animais.

\section{Referências}

Abdel-Ghaffar, F., Al-Quraishy, S., Sobhy, H. \& Semmler, M. (2008). Neem seed extract shampoo, Wash Away Louse®, an effective plant agent against Sarcoptes scabiei mites infesting dogs in Egypt. Parasitol., 104, 145-148.

Adams, R. J., Kim, S. S., Mollenkopf, D. F., Mathys, D. A; Schenemann, G. M, Daniels, J. B. \& Whittum, T. E. (2018). Antimicrobial-resistant Enterobacteriaceae recovered from companion animal and livestock environments. Zoonoses and Public Health., 65(5), $519-527$.

Almeida, L. C., Furtado, G. D. \& Farias, L. A. (2019). Sarna sarcóptica em cães: uma breve revisão. Environmental Smoke, 2(2), $117-121$. 
Araujo, S. A., Silva, O. N. \& Leal, R. D. (2020). One health - a saúde única sob a percepção do estudante de medicina veterinária do Distrito Federal. Revista ciência e saúde animal, 2(2), 9-18.

Arias, M. V. B. \& Carrilho, C. M. D. M. (2012). Resistência antimicrobiana nos animais e no ser humano. Há motivo para preocupação? Semina: Ciências Agrárias, 33(2), 775-790.

Baptista, B. E. (2015). Estudo de estabilidade e eficácia de formulação tópica fitoterápica para tratamento de dermatofitose animal (Tese de doutorado). Universidade Federal de Juiz de Fora, Pós-Graduação em Saúde, Juiz de Fora, Brasil.

Bell, K. L. (2002). Holistic Aromatherapy for Animals: A Comprehensive Guide to the Use of Essential Oils e Hydrosols with Animals. Findhorn: Findhorn Press.

Branco, P. A. C., Soares, R.; Vieites, F., Cabral, N. \& Tavares, E. (2011). Efeitos de óleos essenciais como promotores de crescimento em leitões recém desmamados. Archivos de Zootecnia, 60(231), 699-706.

Canavari, I. C., Hernandez, G. V., Costa, M. T. \& Camplesi, A. C. (2017). Doenças Dermatológicas de Caráter Zoonótico. Investigação, 16(1), $18-24$.

Cleff, B. M., Versteg, N., Gressler, P. M. R., Stelmake, L. L. \& ROSA, S. C. (2020, maio - agosto). Trajetória do projeto de extensão: "Medicina Veterinária na promoção da saúde humana e animal: Ações em comunidades carentes como estratégia para o enfrentamento da desigualdade social". Expressa extensão, 25(2), 80-89.

Cossolosso, D. S. (2013). Atividades leishmanicida e antioxidante dos óleos essenciais de plantas encontradas no Nordeste brasileiro (Dissertação de mestrado). Universidade Estadual do Ceará, Faculdade de Veterinária, Fortaleza, Brasil.

Cavalcanti, E. A. N. L. D., Ignácio, T. C., Kunrath, S. E., Meinerz, A. R. M., Farias, R. O. \& Osório, L. G. (2018, novembro). Esporotricose: Revisão. Pub. Vet., 12(11), 1-5.

Fang, F., Candy, K., Melloul, E., Bernigaud, C., Chai, L., Darmon, C., Durand, R., Botterel, F., Chosidow, O., Izri, A., Huang, W. \& Guillot, J. (2016). In vitro activity of ten essential oils against Sarcoptes scabiei. Parasites \& Vectors, 9:594, 1-7.

Faria, A. M. (2011). Tratamentos convencionais e fitoterápicos para o controle de sarna sarcóptica nos animais domésticos (revisão de literatura) (Dissertação de mestrado). Universidade Federal de Goiás, Pós-Graduação em Ciência Animal, Escola de Veterinária e Zootecnica, Goiânia, Brasil.

Fernandes, V. T. R., Arruda, V. M. A., Oliveira, M. R. V., Queiroz, F. A. J., Melo, S. A., Dias, D. K. F., Marinho, M. B. J., Souza, F. R., Souza, V. O. A. \& Filho, S. A. C. (2015). Aditivos fitopatogênicos na alimentação de frangos de corte: óleos essenciais e especiarias. Pesquisa brasileira veterinária, 9(12), 502557.

Gondim, L. C. L. A. \& Araujo, L. K. A. (2020, janeiro-março). Aspectos clínicos, diagnósticos e terapêuticos da dermatofitose em cães e gatos e sua importância como zoonose. Revista Brasileira de Educação e Saúde, 10(1), 86-94.

Gottlieb, O. R., Kaplan, M. A. C. \& Borim, M. R. M. B. (1996). Biodiversidade: Um enfoque químico-biológico. Rio de Janeiro: Editora da UFRJ.

Gaio, F. C., Lopes, E. S., Lima, B. P., Carmo, C. C., Marques, A. R., Oliveira, F. R., Amaral, M. S. M. G., Pascoal Filho, N. M., Carreira, A. S., Beleza, A. J. F., Teixeira, R. S. C., Havt, A. \& Maciel, W. C. (2019, setembro-outubro). Bactérias zoonóticas isoladas de passeiriformes silvestres recuperados do tráfico de animais no estado do Ceará/Brasil. Arquivo Brasileiro de Medicina Veterinária e Zootecnia, 71(5), 1488-1496.

Hernandez, B. J., Angarita, M. M. \& Prada, Q. C. (2017) Impacto do uso de antimicrobianos na medicina veterinária. Ciencia y agricultura, 14(2), 27-38.

Karkman, A., Do, T. T., Walsh, F. \& Virta, M. P. J. (2018, março). Antibiotic-Resistance Genes in Waste Water. Trends in Microbiology, 26(3), 220-228.

Lima, A. P. L. (2018). Atividade leishmanicida do óleo essencial de Siparuna guianensis e do isolado $\alpha$-Bisabolol isolado de Siparuna guianensis contra Leishmania amazonensis (Dissertação de mestrado). Universidade Federal de Goiás, Pós-Graduação em Ciências Aplicadas à Saúde, Regional Jataí, Jataí, Brasil.

Lima, R. M. (2017). Óleos essenciais como alternativa inovadora para o tratamento da esporotricose (Dissertação de mestrado). Universidade Federal de Juiz de Fora, Faculdade de Medicina, Juiz de Fora, Brasil.

Loureiro, R. J., Roque, F., Rodrigues, A., Therdeiro, M. T. \& Tamareira, E. (2016). O uso de antibióticos e as resistências bacterianas: breves notas sobre a sua evolução. Revista portal saúde pública, 34(1), 77-84.

Lupe, F. A. (2007). Estudo da composição química de óleos essenciais de plantas aromáticas da Amazônia (Dissertação de mestrado). Universidade Estadual de Campinas, Instituto de Química, Campinas, São Paulo, Brasil.

Mateus, W. S. (2016). Avaliação dos efeitos fungistáticos e fungicidas de óleos essenciais em microrganismos causadores de dermatomicoses (Dissertação de mestrado). Universidade Federal do Triângulo Mineiro, Programa de Mestrado Profissional em Inovação Tecnológica, Uberaba.

Nelson, A. (2013). Aromatherapy for parrots: using ancient healing methods in today's companion birds. CreateSpace Independent Publishing Platform.

Neves, J. J., Paulino, O. A., Vieira, G. R., Nishida, K. E. \& Coutinho, A. D. S. (2018, novembro-dezembro). Presença de dermatófitos em pets infectados e em seu ambiente domiciliar. Arquivo Brasileiro de Medicina Veterinária e Zootecnia, 70(6), 1747-1753.

Oliveira, S. \& MORAIS, P. A. C. (2019, setembro). Desenvolvimento de uma emulsão o/a associada ao óleo essencial de gerânio (Pelargonium graveolens) e ao óleo essencial de palmarosa (Cymbopogon martinii). Brazilian Journal of Natural Sciences, 2(3), 127.

Pozzo, M. D., Viegas, J., Santurio, D. F., Rossatto, L., Soares, I. H., Alves, S. H. \& Costa, M. M. (2011, abril). Atividade antimicrobiana de óleos essenciais de condimentos frente a Staphylococcus spp isolados de mastite caprina. Ciência Rural, 41(4), 667-672. 
Research, Society and Development, v. 10, n. 17, e144101724180, 2021

(CC BY 4.0) | ISSN 2525-3409 | DOI: http://dx.doi.org/10.33448/rsd-v10i17.24180

Santos, K. K. A., Rolon, M., Vega, C., Arias, A. R., Costa, J. G. M. \& Coutinho, H. D. M. (2013). Atividade leishmanicida in vitro de Eugenia uniflora e Mormodica charantia. Revista de Ciências Farmacêuticas Básica e Aplicada, 34(1), 47-50.

Soares, E. C. L., Costa, E. P., Silva, L. C. N. \& Araújo, J. M. (2012). Isolamento, Identificação e Atividade Antimicrobiana de Streptomyces sp. UFPEDA 968. Scientia Plena, 8(12), 1-7.

Ueda, J., Tavernaro, R., Marostega, V. \& Pavan, W. (2019, julho). Impacto Ambiental do Descarte de Fármacos e Estudo da Conscientização da População a Respeito do Problema. Revista Ciências do Ambiente On-Line, 5(1), 1-6.

Waller, S. B. (2015). Potencial anti-Sporothrix spp. de plantas da família lamiaceae (Dissertação de mestrado). Universidade Federal do Rio Grande do Sul, Faculdade de Veterinária, Porto Alegre.

Waller, S. B., Madrid, I. M., Cleff, M. B., Santin, R., Freitas, R. A., Meireles, M. C. A. \& Mello, J. R. B. Effects of essential oils of Rosmarinus officinalis Linn. and Origanum vulgare Linn. from different origins on Sporothrix brasiliensis and Sporothrix schenckii complex. Arquivo Brasileiro de Medicina Veterinária e Zootecnia, 68(4), 991-999. 